\title{
CONDICIONES DE VIDA Y PRIVACIDAD COTIDIA- NA DEL CAMPESINADO LEONÉS DE TIERRA DE CAMPOS: LA COMARCA DE SAHAGÚN EN EL SIGLO XVIIII*
}

\author{
Juan Manuel BARTOLOMÉ BARTOLOMÉ \\ Universidad de León
}

\begin{abstract}
RESUMEN: Estudio de las condiciones de vida y la privacidad cotidiana (como viven, se alojan, comen y visten) de las familias campesinas leonesas de Tierra de Campos (Sahagún) en el siglo XVIII, analizando las diferencias en relación con sus niveles de riqueza patrimonial global..

PALABRAS CLAVE: Inventarios, campesinado, León, siglo XVIII, Tierra de Campos, condiciones de vida.

ABSTRACT: This is a study of the living conditions and daily life of rural families from the town of Sahagún in the Tierra de Campos region of León during the eighteenth century, We have included aspects such as way of life, housing, food and dressing. We have also analysed the the differences in relation to their overall inherited wealth.
\end{abstract}

KEYWORDS: Inventories, peasantry, León, eighteenth century, Tierra de Campos, living conditions.

Adentrarnos en la vida cotidiana del campesinado de la Edad Moderna, incluso del siglo XVIII, constituye una tarea muy ardua debido al problema de las fuentes. No obstante, es necesario a nivel historiográfico abordar este tipo de estudios sobre los grupos no elitistas de la población pero en definitiva, no hay que olvidarlo, mayoritarios y menos homogéneos de lo que la visión tradicional historiográfica nos ha legado ${ }^{1}$.

* Comunicación presentada al Congreso Ocio y vida cotidiana en el mundo hispánico. Siglos XVIXVIII, Universidad de Sevilla, 2003.

${ }^{1}$ Esta hetereogeneidad de las comunidades campesinas ha sido señalada por P. Saavedra de tal forma que la desigual distribución de la propiedad y de la posesión de la tierra y los ganados se muestra más acusada en las comarcas de la meseta norte de España y en Tierra de Campos. P. SAAVEDRA, «El campesinado en la España del A. Régimen: algunas consideraciones», en Santiago 
Por ello, tomando como fuente principal de información los inventarios postmortem contenidos en su mayor parte en las partijas de bienes ${ }^{2}$ hemos elegido como ámbito de estudio el campesinado leonés de la comarca de Tierra de Campos de Sahagún en la segunda mitad del siglo XVIII ${ }^{3}$, planteándonos a través de una metodología sobre todo cuantitativa aunque tampoco despreciaremos la cualitativa ${ }^{4}$ los siguientes objetivos: En primer lugar, analizar los niveles de riqueza patrimonial global de dicho campesinado durante la segunda mitad del siglo XVIII y su estructura interna, teniendo muy en cuenta el papel de los bienes mobiliarios sobre los raíces y semovientes. En segundo lugar, adentrarnos en el análisis de la riqueza mobiliaria, su estructura interna y las diferencias observadas en las diferentes familias campesinas según su nivel de riqueza patrimonial global. Y en

CASTILlO y Roberto FERNÁNDEZ (coordinadores), Historia social y ciencias sociales, LLeida, 2001, pp. 225-246.

${ }^{2}$ En total hemos utilizado un total de 60 inventarios postmortem de los cuales la muestra se reduce a 45 con todos sus bienes tasados.

${ }^{3}$ Esta comarca recibe su denominación por ser la más próxima de la provincia de León a las Tierras de Campos castellanas (Palencia) y por tener unas características geográficas (relieve, clima, suelo, vegetación, etc.) y socieconómicas, en principio, muy similares. Así, para mediados del siglo XVIII, según la información suministrada por el Catastro ensenadino, los cultivos principales son el trigal de secano (con el $40,5 \%$ del terrazgo) y el viñedo con un $40 \%$ y su ritmo demográfico va a ser de recuperación en la segunda mitad del setecientos cuando en el resto de la provincia predomina el estancamiento. Ver la Historia de León, Volumen III, Coordinador L. RUBIo PéREZ, León, 1.999.

${ }^{4}$ Una metodología más cualitativa es la que se ha seguido en Francia por M. BAULANT, «Niveau de vie des families rurales dans la Brie du XVIIIe siecle», en R. BonNAIN, G. BoucharD et J. GoY (dir.), Transmetre, heriter, succeder. La reproduction familiale en milieu rurale. France-Quebec, XVIIIe-XXe, París, 1.992, pp. 135 y sgs., consistente en agrupar los objetos en capítulos previamente diseñados y calcular a continuación porcentajes evolutivos de su presencia en los recuentos. También en España con la misma línea metodológica se han abordado trabajos como el realizado para Vitoria de Paloma Manzanos ArReal, «La casa y la vida material en el hogar. Necesidades vitales y niveles de vida en la Vitoria del siglo XVIII», en José Ma ImICOZ BEUNZA (Dir.), La vida cotidiana en Vitoria en la Edad Moderna y contemporánea, Donostia, 1.995, pp.199-236. La metodología elegida cuantitativa se basa fundamentalmente en los valores de las tasaciones en reales que han sido realizadas de los objetos inventariados entrando en juego no sólo la presencia o no de objetos, sino también criterios muy importantes para establecer el nivel de vida como la calidad y el estado de uso de los mismos. En esta línea son muy interesantes los estudios ya realizados sobre el campesinado leonés, lo cual nos permite efectuar comparaciones, de la Vega Baja del Esla de José M. Pérez GARCÍA, «Evolución de los niveles de vida en la Vega del Esla entre 1700 y 1850», en Un modelo social leonés en crecimiento: la vega Baja del Esla entre 1700 y 1850, León, 1.998, pp. 159-170. Y los efectuados por Juan Manuel BARTOLOMÉ BARTOLOMÉ de las comarcas de El Bierzo y Astorga-La Bañeza, Vino y viticultores en el Bierzo. Sociedad y estructuras económicas durante el siglo XVIII León, 1.996.«Condiciones de vida y pautas de consumo del campesinado leonés de la Bañeza y Astorga (1750-1850)», Comunicación presentada a la VII ${ }^{a}$ Reunión Científica de la Fundación Española de Historia Moderna, Ciudad Real, 2.002. 
tercer lugar, abordaremos de forma más pormenorizada, ya que consideramos que constituye el gran objetivo del trabajo que presentamos, las condiciones o estilo de vida del campesinado terracampino y su homogeneidad o polarización según las riquezas patrimoniales globales. Es decir, en definitiva nos proponemos dar respuesta, teniendo siempre presente las limitaciones de la fuente, a aspectos de la privacidad cotidiana: cómo viven, se alojan, comen y visten las familias campesinas de la Edad Moderna.

\section{LA RIQUEZA PATRIMONIAL GLOBAL: LA RELEVANCIA DE LOS BIENES MOBILIARIOS.}

El nivel medio de riqueza patrimonial del campesinado en la segunda mitad del siglo XVIII es de 9.863 reales. Se trata, por lo tanto, de un nivel de riqueza inferior, con más de 3.000 reales de diferencia, del de los campesinos leoneses de la Vega Baja del Esla ${ }^{5}$ e incluso de los de la zona de La Bañeza-Astorga ${ }^{6}$.

El análisis de la estructura interna de la riqueza patrimonial sigue otorgando, al igual que ocurría en los estudios comparativos, el primer puesto a las tierras de labor (tierras de cereal sobre todo de secano y viñedo), sin embargo, la importancia de dichos bienes raíces, con un 31,6\% de la riqueza patrimonial global, es de nuevo más bajo ${ }^{7}$, lo cual quizás pueda ser debido, más que a unas explotaciones agrícolas inferiores, a un menor precio de las tierras ante la mayor oferta del terrazgo productivo y la menor presión ejercida por la población, lo cual significaría unos valores de tasación de dichas tierras en los inventarios más bajos. También destaca la relevancia de los bienes mobiliarios ya que con el $27,7 \%$ de la riqueza patrimonial global se sitúan en un segundo lugar por encima de la valoración de las cabañas ganaderas y de las viviendas y dependencias complementarias ${ }^{8}$. Y finalmente, las viviendas y dependencias complementarias y la ganadería siguen

5 En 1730-59 la media de riqueza patrimonial se sitúa en 13.838 reales y en 1760-89 se incrementa a 21.218 reales. José M. PÉREZ GARCíA, «Evolución de los niveles de vida...», Art. cit., p.161.

${ }^{6}$ En 1750-60 estos niveles medios se sitúan en 13.136 reales para incrementarse también a fines del siglo en 21.031 reales. Juan M. BARTOlOMÉ BARTOLOMÉ, «Condiciones de vida y pautas del consumo...», Art. cit.

${ }^{7}$ En la Vega Baja del Esla oscila entre el 45,5\% y el 43,4\% para la segunda mitad del siglo XVIII y La Bañeza-Astorga entre el 38,2\% y el 46,2\%. Ibidem.

${ }^{8} \mathrm{Su}$ peso en la estructura de la riqueza global es mayor que en los casos de la Vega Baja del Esla y los de la Bañeza-Astorga donde en estas últimas zonas en 1750-60 con un 21,8\% se situaba en tercer lugar, descendiendo al $15,2 \%$ a fines del siglo. Aunque nunca hay que olvidar que sus medias familiares, 2.727 reales, siguen estando por debajo. Ibidem. 
ocupando un lugar destacado y con porcentajes, un $23,4 \%$ y un $17,3 \%$ respectivamente de la estructura global patrimonial, ya más similares a los comparativos leoneses. Esta importancia de las casas y viviendas complementarias se debe a los elevados porcentajes de posesión de casas y al alto valor de tasación de las mismas, ya que incluso entre el campesinado más pobre, por debajo de los 1.500 reales de riqueza patrimonial, es frecuente encontrar casas tasadas entre 250 y 440 reales 9 . Aunque los inventarios postmorten son muy parcos en la información cualitativa si que podemos señalar que se trata de casas donde los niveles de riqueza establecen diferencias entre los campesinos más pudientes con casas de varios pisos y habitaciones espaciosas, divididas por tabiques de obra o cortinaje y las casas de lo más modestos o pobres, de un solo piso, con ventanas pequeñas para mantener el calor en invierno y preservarse de él en verano y estancias más pequeñas donde el hogar o cocina ocupaba un lugar preferencial, a veces único, no sólo para comer sino también para habitar y descansar (dormir junto al fuego) ${ }^{10}$. Además, es frecuente, lo cual también explica su alta tasación, que unido al hogar y cuartos de residencia estén también los edificios o estancias del corral, las caballerizas, los pajares, la bodega y a veces también el lagar ${ }^{11}$. Casas, en definitiva, construidas, principalmente las más modestas, a base de una estructura de madera que era rellenada con tapial (mezcla de barro y paja), ya que hasta el siglo XIX no se generalizan las casas de piedra o ladrillo ${ }^{12}$.

En conclusión, se trata de un campesinado con unos niveles medios de riqueza patrimonial por debajo de sus homólogos leoneses ${ }^{13}$ y muy heterogéneo, como lo demuestra el hecho de que únicamente el $42,2 \%$ de la muestra se sitúan entre el

${ }^{9}$ Es lo que sucede, por ejemplo, con Manuel de la Fragua, vecino de San Pedro de Dueñas, el cual tiene una casa valorada en 440 reales, casi la mitad de su escaso patrimonio global de 937 reales. Archivo Histórico Provincial de León (A.H.P.L.), Juan Felipe y Godoy, Caja 4461.

${ }^{10}$ Al igual que ocurría en Vitoria, P. MANZANOS ARREAL, «La casa y la vida material.....», Art. cit., pp. 205, 206.

${ }^{11}$ Por ejemplo, uno de los ricos campesinos con un valor patrimonial de 37.138 reales, Manuel Prieto, vecino de Sahagún, posee tres casas valoradas en 8.500 reales y todas ellas constan de cuartos altos y bajos, corral, caballerías, pajares y bodega. A.H.P.L., Juan Felipe y Godoy, Caja 4459.

12 Bartolomé Yun CASAlilla, «Peasant material culture in Castile (1750-1900): some proposals», en A. SCHUURMAN Y L. WALSH (eds.), 1994, pp.127-130

13 Exceptuando el campesinado de El Bierzo donde en su zona de economía más vitícola, El Bierzo Bajo, no llegan a los 2.000 reales. Juan M. BARTOLOMÉ BARTOLOMÉ, Vino y viticultores..., Op. cit., p. 211. 
umbral de riqueza global de 1.500 reales y 5.000 reales $^{14}$, distribuyéndose el resto entre $4,5 \%$ por debajo de los 1.500 reales, el $22,2 \%$ de 5.000 a 10.000 reales, el $22 \%$ de 10.000 a 20.000 reales y el $8,9 \%$ por encima de los 20.000 reales.

\section{LA RIQUEZA MOBILIARIA: LA IMPORTANCIA DE LOS PRO- DUCTOS AGRARIOS Y DE LA DESPENSA}

Los bienes mobiliarios suponían un $27,7 \%$ del total de la riqueza patrimonial de nuestras familias campesinas, sin embargo, la media global de dichos bienes, 2.727 reales exceptuando los animales domésticos, estaría de nuevo por debajo, aunque las diferencias sean más cortas, de los campesinos de la Vega Baja del Esla, La Bañeza y Astorga ${ }^{15}$. Si descendemos a un estudio más minucioso de la estructura interna de la riqueza mobiliaria podemos apreciar las siguientes características: Por una parte, el escaso peso de los aperos, útiles de labranza, con un 14,2\% y 389 reales de media ${ }^{16} \mathrm{y}$ del dinero en efectivo, créditos a favor y alhajas con un $7,7 \%$ y tan sólo 839 reales de media ${ }^{17}$. Por otra parte, los que realmente sobresalen, con un $47,3 \%$, de la estructura de los bienes mobiliarios son los productos agrarios de la despensa ${ }^{18}$, lo cual nos indica una alta capacidad de producción agraria y una plenitud en las despensas campesinas terracampinas en la segunda mitad del siglo XVIII. Además, la relación de productos agrarios que figuran en los inventarios nos permite acercarnos, aunque sea tímidamente, a la comida de los campesinos. Así, más que carne de aves o de vacuno - sólo de forma muy esporádica aparece la cecina- u ovino lo normal era encontrar en dichas despensas productos derivados

14 Los cuales se pueden catalogar como patrimonios modestos, ya que poseen explotaciones agrarias dedicadas al cultivo del viñedo y cereal, vivienda y dependencias complementarias y una cabaña ganadera complementaria interesante. Es el caso, por ejemplo, de Manuel Conde Guaza, vecino de Sahagún, con un valor patrimonial global de 4.200 reales, que explota tierras de viñedo y cereal tasadas en 1.895 reales, tiene una casa valorada en 400 reales y una cabaña ganadera en 435 , consistente en un buey tasado en 250 reales, una cerda en 95 reales y una pollina en 90 reales.. A.H.P.L., Juan Felipe y Godoy, Caja 4.459.

${ }^{15}$ En la Vega Baja del Esla en 1760/89 la media es 4.517 reales, pero en las zonas de Astorga-La Bañeza en 1750-60 la media es de 2.868 reales y en 1790-18000 de 3.184. José M. PÉREz GARCíA, «Evolución de los niveles de vida...», Art. cit., p. 161. Juan M. Bartolomé Bartolomé, «Condiciones de vida...», Art. cit.

${ }^{16}$ Inferior a los estudios comparativos leoneses. Ibidem.

${ }^{17}$ En este capítulo las diferencias no son tan apreciables con los casos comparativos. Ibidem.

${ }^{18}$ Con 1.289 reales de media están por encima de las medias halladas en Astorga-La Bañeza y las de los años 1730-59 de la Vega Baja del Esla, siendo de nuevo superiores las de los años 1760/89. Ibidem. 
del cerdo (manteca, tocino, etc. $)^{19}$. También aparece la harina aunque no especifica de que tipo de cereal, lo cual nos puede hacer pensar que el pan que consumían podría ser más que de trigo, destinado a pagar las rentas, de centeno, o de avena ${ }^{20}$. En el puchero diario no faltaban las legumbres tradicionales -titos, garbanzos, lentejas- no obstante, no encontramos en los inventarios las alubias ${ }^{21}$ y tampoco figuran las patatas, aunque es probable que ya se sembrase este tubérculo de forma muy selectiva y no se declárese debido a ser un cultivo noval. Finamente esta pobre dieta campesina era acompañada con el consumo de abundantes cantidades de vino, más en una zona donde la producción era significativa. No podemos proporcionar datos concretos del consumo, pero dada la tónica general europea estaría por encima del litro diario por persona adulta ${ }^{22}$. Por último, hay que destacar que los bienes del ajuar, mobiliario de la casa, útiles domésticos, cuadros, libros, se sitúan en un segundo lugar de la riqueza mobiliaria con un 30,8\% y 839 reales de media $^{23}$. Este tipo de bienes son los que, por otra parte, nos dan a conocer las condiciones y el estilo de vida de la población campesina.

\section{LAS CONDICIONES O ESTILO DE VIDA: EL MOBILIARIO, LA DECORACIÓN, LA ROPA Y LOS ARTÍCULOS DE LUJO}

Con el objetivo de poder aproximarnos al conocimiento de las condiciones o estilo de vida de las familias campesinas hemos desglosado los bienes del ajuar, mobiliario, etc. en los siguientes capítulos: decoración de la casa (objetos religiosos), mobiliario de la casa, útiles cocina (menaje, loza fina, etc.), ropa personal, ropa de cama, ropa de casa, libros y joyas. Además del estudio

19 Las aves se consideraban en la Edad Moderna alimentos propios de príncipes y reyes. En cambio la carne del cerdo que hoza en el barro o los nabos que crecen bajo tierra se consideraban alimentos propios de vulgares campesinos. Raffaella SARTI, Vida en familia, casa, comida y vestido en la Europa Moderna, Barcelona, 2.002, p. 219.

${ }^{20} \mathrm{Al}$ igual que sucedía en el resto de Europa. Ibidem, p.221.

${ }^{21}$ Las cuales se sumaron rápidamente a las legumbres conocidas tradicionalmente en Europa y con una aportación proteica a la dieta de los pobres muy importante. Ibidem, p. 231.

${ }^{22}$ El consumo del vino y cerveza estaba muy generalizado debido a varias razones: completaba el aporte calórico de la dieta, era difícil obtener agua potable, la creencia de que el agua era mala para la salud, la necesidad de facilitar la masticación y deglución del pan duro, el consumo de alimentos muy salados, y finalmente, permitía alcanzar un estado de euforia y excitación necesario para afrontar las inseguridades, los temores y las fatigas de la vida diaria, para ahuyentar el espectro o los retortijones del hambre. Ibidem, pp. 238, 239.

${ }^{23}$ Porcentaje y media inferiores a las del campesinado de la Vega Baja del Esla -sobre todo en la muestra de los años 1760/89- y al de la La Bañeza-Astorga. José M. PÉREZ GARCíA, «Evolución de los niveles...», art, cit., p. 161. Juan M. BARTOLOMÉ BARTOLOMÉ, «Condiciones de vida...», Art. cit. 
cuantitativo nos adentraremos también en los aspectos cualitativos y trataremos de analizar las diferencias de acuerdo con la riqueza patrimonial global de las familias campesinas.

La estructura interna de este tipo de bienes mobiliarios es a primera vista muy similar a la del campesinado leonés de la Vega Baja del Esla, La Bañeza-Astorga, pero un estudio más profundo deja traslucir aspectos diferenciales: en primer lugar, la media de estos bienes, con 839 reales, es bastante modesta y en general está por debajo de la de los demás campesinos leoneses ${ }^{24}$. En segundo lugar, la relevancia de los textiles -ropa personal, de cama y casa- es todavía más fuerte, con un $65,3 \%$ del valor de tasación de este tipo de bienes, que en el resto del campesinado leonés. En tercer lugar, dentro de este gran peso de los textiles sigue ostentando el lugar preferente -al igual que ocurre con el resto del campesinado leonés- el calzado y la ropa personal, pero la ropa de cama adquiere una importancia, con un $28 \%$, no conocida en los anteriores estudios comparativos, hasta el punto de que relega a un tercer puesto al mobiliario de la casa con un 19,2\%. En cuarto lugar, los objetos de decoración de la casa, que serán sobre todo religiosos, sólo serán valorados en una media de 25 reales $^{25}$. Y finalmente, el carácter más modesto de las condiciones de vida de este campesinado terracampino se vuelve a remarcar en la ausencia total de libros inventariados y en la escasa presencia de alhajas y objetos de oro y plata bien para uso personal o bien como cubertería ${ }^{26}$.

Ahora bien, las diferencias no sólo se aprecian en la visión genérica efectuada sino también y con mayor riqueza en un estudio más detallado. Así, ya hemos destacado la gran relevancia en la valoración, superando en la misma a los casos comparativos leoneses, del stock de productos textiles. Y dentro de los mismos como es el calzado y la ropa destinada a la vestimenta personal (interior y exterior)

\footnotetext{
${ }^{24}$ Nos referimos a los mencionados de la Vega Baja del Esla y los de la zona La Bañeza-Astorga. Únicamente los campesinos de El Bierzo Bajo tendrán unas medias más bajas con 744 reales pero para todo el siglo XVIII. José M. PÉrez García, «Evolución de los niveles de vida...», Art. cit., p.167. Juan M. BARTOlomé BARTOlomé, «Condiciones de vida...», Art. cit. Vino y viticultores..., Op. cit. p.364.

${ }^{25}$ Cantidades muy inferiores a los 308 reales de media de la zona de La Bañeza-Astorga. Juan M. BARTOlOMÉ BARTOlOmé, «Condiciones de vida...», Art. cit.

${ }^{26}$ Con el $0,3 \%$ y una media de tan sólo 2,5 reales. En cambio en el campesinado de La Bañeza y Astorga este tipo de bienes adquirieron una gran importancia en el siglo XVIII con medias de 135 reales ya que se consideraban no sólo un distintivo de riqueza sino también una inversión segura ante las oscilaciones y devaluaciones monetarias. Ibidem.
} 
la mejor tasada, con 295 reales de media ${ }^{27}$, pero lo más novedoso es la importancia que adquiere la ropa de cama con 235 reales de media ${ }^{28}$. Aún podemos profundizar más si introducimos como nueva variable de análisis los niveles de patrimonio global del campesinado y la relación de los textiles con los mismos. De ese modo, las familias campesinas con los valores patrimoniales más bajos, inferiores a los 1.500 reales, su calzado y ropa personal es valorada en 165 reales de media, lo cual supone con el $61,3 \%$ la cantidad más alta de este tipo de bienes, y en cambio la ropa de casa en tan sólo 25,5 reales de media, lo que significa un $9,5 \%$ de sus bienes mobiliarios analizados en este apartado ${ }^{29}$. En cambio, según va subiendo el nivel patrimonial global desciende la importancia porcentual del calzado y la ropa personal, aunque sigue ostentando el primer puesto, y se incrementa la de la ropa de casa y particularmente la de cama, hasta el punto de que en los patrimonios más solvente, por encima de los 20.000 reales la ropa personal significa el $21,8 \%$ de la estructura de los bienes y la ropa de cama el 33,3\% y una media de 608 reales frente los 397 reales de media de la ropa personal ${ }^{30}$.

Por lo tanto, se observan grandes contrates entre el propio campesinado en cuanto a la abundancia de piezas y calidad de las mismas de la ropa de casa y cama y sobre todo de la ropa destinada a vestir el cuerpo. Diferencias que, en definitiva, como hemos apreciado anteriormente, guardan relación con el nivel de riqueza patrimonial global. De todas formas, sí podemos acercarnos al modelo genérico de

${ }^{27}$ Aunque tenemos que precisar que no es extraño que en los inventarios no encontremos ningún elemento del vestir diario, ya que el difunto/a se llevaría a la tumba el único traje que poseía, sin embargo, la media también es inferior a la de La Bañeza-Astorga de 404 reales. Ibidem.

${ }^{28}$ Superior a los 163 reales de los campesinos de la zona de La Bañeza y Astorga. Ibidem.

${ }^{29}$ Es el caso, por ejemplo, de Manuel de la Fragua, vecino de San Pedro de Dueñas, con un valor patrimonial total de 937 reales, que posee una ropa personal tasada en 114 reales (consistente en una capa, dos anguarinas, jubón, ropilla, casaca, dos manteos, un rebociño y un par de zapatos) y en cambio la ropa de cama es más elemental y de ahí que tan sólo esté valorada en 33 reales (un jergón, una manta, y dos almohadas). A.H.P.L., Juan Felipe y Godoy, Caja 4461

${ }^{30}$ Desequilibrio que en realidad no es tan fuerte debido a que en algunos inventarios de esta muestra no aparece ni se tasa la ropa personal. Aspecto al que no encontramos una explicación razonable, ya que debido a su alto nivel económico de los difuntos es difícil que sólo posean la ropa personal con la que son enterrados o que ésta sea previamente vendida al inventario para recaudar dinero por el viudo/a De todas formas, como ejemplo tenemos el de Domingo Herrero Rojo, vecino de Calzada del Coto, con un valor patrimonial de 47.919 reales, cuya tasación de la ropa personal, con 572 reales (donde figuran mayor cantidad y calidad de las prendas de vestir, como capas de paño de Astudillo, etc.), se ve superada por la ropa de cama con 596 reales (once mantas de lana generalmente casera, cinco cobertores -dos de ellos de Palencia-, dos sábanas, tres almohadas, una colcha de gusanillo pintada, etc.). Y finalmente, también encontramos ropa de casa (tres tablas de manteles, cuatro servilletas) valorada en 53 reales. Id., Caja 4460. 
vestimenta tanto de los hombres como de las mujeres. Así, en los varones como ropa interior encontramos las camisas ${ }^{31}$ de tejidos bastos como el lienzo. Y como ropa exterior: los calzones de paño, jubones de estameña, anguarinas de paño, capas de paño, medias y zapatos de baqueta. En la mujeres, como ropa interior: calzones y enaguas y también camisas, generalmente de lienzo (estopa) y como externa: justillos, guardapiés, basquiñas, mantilla, jubones sin mangas, cuerpos, manteos, almillas, casacas, delantales, mantillas y para cubrir la cabeza: pañuelos de lienzo o seda. Todas las prendas están elaboradas utilizando en general como fibra textil el lino o la lana (estameña) ya que la más novedosa y relevante de cara a la modificación de las pautas de consumo: el algodón, no logrará abrirse hueco en estos medios rurales hasta la década de los años 30 del siglo XIX ${ }^{32}$.

Para concluir en este apartado de la ropa también resulta interesante remarcar que la ropa de casa empieza a figurar y se incrementa en el número de piezas y en calidad, por encima de los valores bajos de la media, particularmente en las familias campesinas de mayor riqueza patrimonial. Así, en sus ajuares encontramos servilletas (algunas de gusanillo), manteles (también de gusanillo), objetos de aseo: paños de manos y también artículos decorativos: las cortinas (generalmente de lienzo, o las más frecuentes de bayeta verde). Todo esto denota una mayor predilección por la comodidad y el lujo en el quehacer cotidiano.

En cuanto al mobiliario de la casa es desplazado, como ya hemos señalado, de su segundo puesto, como se apreciaba en los estudios comparativos de sus homólogos leoneses, a un tercer lugar con un $19,2 \%$ y con un media por familia campesina de tan sólo 161 reales $^{33}$. De nuevo se vuelven a apreciar diferencias

${ }^{31}$ El uso de ropa interior, de lino o estopilla -el algodón tardará en introducirse hasta los años 30 del siglo XIX- es muy interesante de cara a la higiene personal consiguiendo reemplazar al agua debido a que cambiarse de camisa equivalía a nuestra ducha. Paloma MANZANAS A., «La casa y la vida...», Art. cit., p.226. No aparecen, en cambio, los calzoncillos, únicamente hemos hallado tres pares de los mismos tasados en 18 reales en el inventario de Josefa Rodríguez, A.H.P.L., Antonio Mata, Caja 4464.

32 Sólo en algunos inventarios, como el de Marcela Calvo, en 1776, aparece un pañuelo de algodón. AH.P.1., Antonio Mata, Caja 4461. Para una mayor información sobre las pautas de consumo y sus mutaciones sobre los productos textiles a partir de los años 30 del siglo XIX ver trabajos en España del grupo de investigación dirigido por el Dr. Bartolomé Yun CASAliLla, Consumo y comercialización de textiles en Castilla y Cantabria, 1750-1914, DGICYT y en particular el citado de Juan M. BARTOlOMÉ BARTOlOMÉ, «Condiciones de vida...», Art. cit.

${ }^{33}$ Cuando en la zona de Astorga-La Bañeza es de 207 para los años finales del siglo XVIII y sobre todo en la Vega Baja del Esla es de 298,4 para los años 1760-89. Sólo el campesinado de El Bierzo Bajo tiene valores inferiores de 111 reales pero para todo el siglo XVIII. José M. PÉREZ 
significativas entre las familias campesinas, las cuales vuelven a guardar una estrecha relación con los niveles de riqueza patrimonial global. De este modo, los campesinos más pobres, por debajo de los 1.500 reales de riqueza patrimonial, sólo poseen unos bienes muebles, valorados en 53 reales de media, que quedan reducidos a la mínima expresión: algunas mesas y sillas, alguna cama acompañada de escasos bancos y escaños situados cerca del fuego para poder también utilizarlos como lugar donde descansar o dormir y finalmente, algunas arcas para guardar desde productos de la cosecha, cereales, harina, hasta todo tipo de objetos variados $^{34}$. Únicamente, los campesinos que podemos catalogar como medios, a partir de los 5.000 reales de riqueza patrimonial, superan la barrera de los 182 reales en mobiliario, lo cual indica mayor cantidad de piezas y de mayor calidad en la materia prima, más que mayor variedad ${ }^{35}$ y las familias campesinas con mayor riqueza patrimonial, más de 20.000 reales, consiguen amueblar sus estancias de la casa con un número de piezas mayor, de mejor calidad de la madera y sobre todo de mayor variedad, tasándose en una media de 409,5 reales $^{36}$.

El menaje de la casa (loza fina, etc.) y los útiles de cocina (donde se incluyen también las calderas) ocupan el cuarto lugar de este tipo de bienes que estamos analizando con un $12,2 \%$. Sus valores medios familiares, 103 reales, se sitúan a nivel comparativo con sus homólogos leoneses por debajo de nuevo de los de la Vega Baja del Esla, pero muy por encima de los de la zona de Astorga-La

García, «Evolución de los niveles...», Art. cit., p.265. Juan M. BArtolomé Bartolomé, «Condiciones de vida....», Art. cit. Vino y viticultores..., Op. cit., p.364.

${ }^{34}$ Por ejemplo, Félix de la Fragua, vecino de la Grañeras, con unos bienes patrimoniales de tan sólo 786 reales, dispone únicamente de una mesa pequeña, tasada en tres reales, un arca grande valorada en 20 reales, dos bancos en tres reales y una cama de tablas viejas en ocho reales. Lógicamente, ello guarda relación con su casa de un solo piso donde hay algún cuarto pero donde sobre todo la pieza más importante es la cocina. Este panorama tan pobre es el mismo que se dibuja para Vitoria. A.H.P.L., Juan Felipe y Godoy, Caja 4463. Paloma MANZANOS ARrEAL, «La casa y la vida material...», Art. cit., pp.212 - 214.

${ }^{35}$ Es el caso de Manuel del Corral, con 5.820 reales de media patrimonial, que posee seis arcas (dos grandes de nogal tasadas en ochenta y cuatro reales), dos escaños, cuatro bancos de respaldo, dos mesas, una cama torneada de haya tasada en 16 reales . A.H.P.L., Antonio Mata, Caja 4460.

${ }^{36}$ Aquí es donde junto a los anteriores muebles, pero más abundantes y de mayor calidad en la madera, nogal frente a la haya, etc., aparecen otros decorando las estancias de las casa y que son signo de distinción y ostentación social. Así, en el inventario de Manuela de Saldaña, con una riqueza patrimonial de 81.146 reales y una tasación de los muebles de 705 reales, figuran piezas distintas destinadas a lograr una mejor decoración de las estancias y a su vez símbolo de una mayor ostentación: un velador de nogal, un bufete grande también de nogal valorado en cincuenta reales y un escritorio de nogal con ocho gavetas y sus portezuelas tasado en setenta reales. Ibidem. 
Bañeza $^{37}$. No obstante, en este tipo de bienes tan básicos para las economías familiares también se apreciarán diferencias entre las familias campesinas pobres, por debajo de los 1.500 reales, donde únicamente poseerán los útiles más elementales de la cocina ${ }^{38}$, y las familias que superan la media de los 103 reales, pero aún así los contrastes son menores que en los anteriores bienes analizados, ya que es cierto que los útiles tradicionales se convierten en más abundantes y de mejor calidad (calderas, cazos, sartenes, trébedes, etc., elaborados con cerámica de Talavera), sin embargo, no aparecerán de forma generalizada los nuevos instrumentos de asar la comida ( asadores, parrillas, espeteras, etc.) ${ }^{39}$ y tampoco abundarán en las familias campesinas de mayor nivel económico los útiles de cocina más vanguardistas y que denotan unas prácticas en la comida más individualizada (platos, vasos y sobre todo los tenedores) ${ }^{40}$.

Si nos adentramos en la decoración de las casas observamos las siguientes características: En primer lugar, un gran empobrecimiento, con tan sólo 25 reales de media, en relación con sus homólogos leoneses de la zona de La BañezaAstorga $^{41}$. En segundo lugar, dentro de ese ambiente de escasez de objetos de adorno se aprecian de nuevo fuertes contrastes entre las familias campesinas más

${ }^{37}$ En la Vega Baja del Esla en el corte cronológico de 1760/89 el nivel medio es casi el doble, 191,6 reales, en cambio, en la zona de Astorga-La Bañeza no superan los 30 reales a finales del siglo XVIII. Ver José M. Pérez García, «Evolución de los niveles de vida...», Art. cit., p.165. Juan M. BARTOlOMÉ BARTOlOMÉ, "Condiciones de vida...», Art. cit.

38 Manuel de la Fragua, vecino de San Pedro de Dueñas, tiene una riqueza patrimonial de sólo 937 reales y su menaje y útiles de cocina que figuran en el inventario son únicamente: una caldera, una sartén, un cazo de azofar, tasado todo en 23 reales. A.H.P.L., Juan Felipe y Godoy, Caja 4461.

39 La aparición de estos útiles es muy relevante para poder estudiar los cambios en la dieta alimenticia de los hombres del A. Régimen, ya que la técnica más usada era la de hervir los alimentos en una olla o caldera, poniéndola directamente sobre las brasas, que solían estar situadas en el centro de la cocina ,o sobre unas trébedes. Sin embargo, al construirse los hogares, a partir del siglo XVIII, contra la pared se abría la posibilidad de elevar los cacharros de cocina por encima del fuego y la instalación de asadores de hierro fundido con lo que se podían asar mejor los alimentos en vez de hervirlos. Paloma MANZANOS ARreal, «La casa y la vida material...», Art. cit., pp. 206-221.

${ }^{40}$ Sí que aparecen chocolateras, y vasos pero no hemos encontrado tenedores. El tenedor se hizo habitual a mediados del siglo XVIII dentro de la tendencia que imponían los manuales de urbanidad de prohibir el uso de los dedos y potenciar más el individualismo. Además, el chocolate, aparte de su valor dietético se convierte también en una bebida indispensable de las reuniones sociales, donde las señoras y sus amigas que acuden a visitarla charlan, mientras saborean el manjar del chocolate. Ibidem, p.222.

${ }^{41}$ En estas familias campesinas las medias oscilan entre los 259 reales de los años 1750-60 y los 357 reales de los años 1790-1800. Juan M. BARtolomé Bartolomé, «Condiciones de vida...», Art. cit. 
modestas, por debajo de los 5.000 reales, las cuales, no olvidemos, casi constituyen la mitad del campesinado con el $46,7 \%$ de la muestra, y las más ricas, por encima de los 20.000 reales. Así, en las primeras, lo más frecuente es que estos objetos decorativos, hasta los más frecuentes religiosos, brillen por su ausencia ${ }^{42}$. En cambio, en las segundas, las estancias son decoradas no sólo con los frecuentes cuadros de temática religiosa sino también con espejos en las paredes y velones ${ }^{43}$. $Y$ finalmente, al igual que ocurría con otros grupos sociales de mayor nivel económico hay un predominio de los cuadros y estampas de carácter religioso ${ }^{44}$.

También si establecemos la comparación con los familias campesinas de La Bañeza y Astorga nos sorprende la escasa presencia de las joyas de uso personal o cubertería $^{45}$. Y finalmente, tampoco encontramos ningún tipo de libro en los inventarios de las familias campesinas terracampinas.

En definitiva, como conclusiones generales podemos señalar las siguientes: Por una parte, se trata de unas familias campesinas donde los niveles de riqueza patrimonial global son en general inferiores a sus homólogos leoneses, lo cual determina el menor peso de las tierras de labor en dicha riqueza y la relevancia de

42 Sólo en un número muy pequeño de familias aparecen cuadros y estampas religisosas colgadas de las paredes. Los ejemplos más extremos son los de Lorenzo Martínez, vecino de Sahagún, que con una riqueza patrimonial de 3.244 resales, en su inventarios aparecen 20 cuadros, tasados en 81 reales, por encima del mobiliario de la casa, útiles de cocina y la ropa de la casa. Cuadros de temática preferentemente religiosa -Santa Marta, Santa Gertrudis, la Peregrina, La Magdalena, etc.- y dos de la calle de Santiago. Y Juana Santos, vecina también de Sahagún, que posee 24 cuadros valorados en 92 reales. A.H.P.L., Juan Felipe y Godoy, Caja 4461.

${ }^{43}$ Es lo que sucede con las estancias de Manuel Prieto, vecino de Sahagún, que tiene junto a los cuadros "un espejo mediano de los de Bilbao en veinte reales" y "un velón de alquimia mediano del cuatro mecheros en treinta reales". Id., Caja 4459.

44 Apreciado también no sólo entre el campesinado sino con mayor profusión entre la burguesía llegando, por ejemplo en la burguesía compostelana, a convertir sus estancias en museos de arte sacro. A. EIRAS ROEL, «La burguesía mercantil compostelana a mediados del siglo XVIII: mentalidad tradicional e inmovilismo económico», en Historia social de Galicia en sus fuentes de protocolos, Santiago, 1.980. Juan M. BARTOlOMÉ BARTOlOMÉ, «Condiciones de vida y pautas de consumo de la burguesía bañezana (1750-1850)» (Artículo en prensa).

${ }^{45}$ Mientras en las zonas de Astorga-La Bañeza los valores medios entre 151 y 184 reales en la segunda mitad del siglo XVIII. En cambio en las familias campesinas de Tierra de Campos, Sahagún y su zona, la media es de tan sólo 2,5 reales, la mayoría del campesinado no posee ningún tipo de joyas o alhajas, por debajo de los 10.00 reales de riqueza patrimonial, y únicamente por encima de los mencionados 10.000 reales figuran en los inventarios este tipo de bienes más suntuoso y de ostentación, pero de forma muy simbólica (una medalla de plata, un barquillo de plata), con 7,3 reales y 10 reales de media en los campesinos entre 10.000 y 19.999 reales y más de 20.000 reales respectivamente. A.H.P.1. (Protocolos Notariales de Sahagún). 
los bienes mobiliarios. No obstante, por otra parte, esa riqueza mobiliaria de nuevo muestra un carácter más agrario, con un papel muy destacado de los productos agrarios de la despensa, lo cual se traduce a su vez en unas condiciones o estilo de vida más precarias que sus convecinos leoneses, sobre todo los de la Vega Baja del Esla y los de la zona Astorga-La Bañeza. De este modo, son los productos textiles, particularmente la ropa destinada a la vestimenta personal y la de cama - con valores más altos en el último caso que los comparativos- más que a la de casa, los que eclipsan a los demás bienes, especialmente a los más suntuosos: los destinados a la decoración de la estancias de la casa y a las joyas y alhajas bien de la cubertería o de uso personal. Y finalmente, al adentrarnos en la privacidad cotidiana de estas familias campesinas de Tierra de Campos, se aprecia una fuerte divergencia entre las mismas, que guarda una relación muy directa con el grado de riqueza patrimonial global. De ahí, que esta diversidad del campesinado no se traduzca únicamente en el tamaño de las explotaciones agrarias y de su cabaña ganadera, sino también en sus condiciones y estilo de vida, en definitiva, en su privacidad cotidiana, orientada a una mayor comodidad y lujo, imitando lógicamente a los grupos sociales superiores sobre todo urbanos ${ }^{46}$, en las familias campesinas de mayor nivel económico.

\footnotetext{
${ }^{46}$ Según conocemos tanto a nivel europeo como en España estos procesos de emulación en las formas de vida, sobre todo las nuevas modas en la vestimenta, nuevas fibras textiles, etc., se producen de las zonas urbanas a las rurales y de los grupos de mayor categoría social y económica a los inferiores. Ver estudios sobre este apasionante tema del consumo de productos textiles a nivel internacional para Inglaterra de N. MCKENDRICK, «The Consumer Revolution of Eighteenth-Century England» en The Birth of a Consumer Society. The Comemmercialization of Eighteenth-Century England, Londres, 1.982. P BORSAY, The English Urban Renaissance. Culture and Society in the Provincial Town 1660-1770, Oxford, 1.989. C. Shammas, The Pre-industrial Consumer in England and America. Oxford, 1.990. L WeATHERILL, Consumer Behaviour and Material Culture in Britain, 1660-1760, Londres-New York, 1.988. Para Francia de D. Roche, La culture des apparences. Une histoire du vêtement XIII-XVIII siècle, Fayard, 1.989. Y en España los trabajos para Cantabria, Castilla y León del grupo de investigación ya citado coordinado por el Dr. Bartolomé YUN Casalilla.
} 
Cuadro 1: Riqueza patrimonial global de las famílias campesinas (en reales y porcentaje sobre el valor del stock inventariado de bienes)

\begin{tabular}{lcc}
\hline \multicolumn{1}{c}{ Bienes } & Reales & $\%$ \\
\hline Tierras de labor & 140.500 & 31,6 \\
Vivienda y dependencias complementarias & 104.110 & 23,4 \\
Ganadería & 76.499 & 17,3 \\
Bienes mobiliarios & 122.709 & 27.7 \\
\hline Total & 443.818 & 100 \\
\hline
\end{tabular}

FUENTE: A.H.P.L. (45 inventarios con los bienes tasados).

Cuadro 2: Distribución riqueza patrimonial mobiliaria de las familias campesinas (en reales y porcentaje sobre el valor del stock inventariado de bienes).

\begin{tabular}{lcc}
\hline \multicolumn{1}{c}{ Bienes } & Reales & $\%$ \\
\hline Aperos, útiles de labranza, cubas, etc. & 17.486 & 14,2 \\
Productos agrarios de la despensa (fibras textiles, etc) & 58.002 & 47,3 \\
Créditos, deudas a favor, dineros y alhajas & 9.449 & 7,7 \\
Ajuar, mobiliario, útiles domésticos, cuadros, libros & 37.772 & 30,8 \\
\hline Total & 122.709 & 100 \\
\hline
\end{tabular}

FUENTE: A.H.P.L. (45 inventarios con los bienes tasados ).

Cuadro 3: Riqueza mobiliaria. Valores medios familiares.

\begin{tabular}{lc}
\hline \multicolumn{1}{c}{ Bienes } & Reales \\
\hline Aperos, útiles de labranza, cubas, etc. & 389 \\
Productos agrarios de la despensa (fibras textiles, etc) & 1.289 \\
Créditos, deudas a favor, dineros y alhajas & 210 \\
Ajuar, mobiliario, útiles domésticos, cuadros, libros & 839 \\
\hline Total & 2.727 \\
\hline
\end{tabular}

FUENTE: A.H.P.L. (45 inventarios con los bienes tasados).

Cuadro 4: Condiciones o estilo de vida de las familias campesinas (en reales y porcentajes sobre el valor del stock inventariado de bienes).

\begin{tabular}{lcc}
\multicolumn{1}{c}{ Bienes } & Reales & $\%$ \\
\hline Decoración de la casa (objetos religiosos) & 1.125 & 3 \\
Mobiliario de la casa & 7.245 & 19,2 \\
Útiles cocina (menaje, loza fina, etc.) & 4.614 & 12,2 \\
Ropa personal & 13.295 & 35,3 \\
Ropa de cama & 10.566 & 28 \\
Ropa de casa & 785 & 2 \\
Joyas & 114 & 0,3 \\
Libros & 0 & 0 \\
\hline Total & 37.744 & 100 \\
\hline
\end{tabular}

FUENTE: A.H.P.L. (45 inventarios con los bienes tasados) 
Condiciones de vida y privacidad cotidiana del campesinado leonés de Tierra de Campos: la comarca...

Cuadro 5: Condiciones o estilo de vida según los niveles de riqueza patrimonial global (en reales y porcentaje sobre el valor del stock de bienes inventariado).

\begin{tabular}{lcccccccccccccc}
\hline \multirow{2}{*}{ Reales } & \multicolumn{2}{c}{$\begin{array}{c}\text { Decoración } \\
\text { de la casa }\end{array}$} & \multicolumn{2}{c}{ Mobiliário de } & \multicolumn{2}{c}{$\begin{array}{c}\text { Utiles cocina, } \\
\text { la casa }\end{array}$} & \multicolumn{2}{c}{$\begin{array}{c}\text { Ropa } \\
\text { etc. }\end{array}$} & \multicolumn{2}{c}{$\begin{array}{c}\text { Ropa de } \\
\text { personal }\end{array}$} & \multicolumn{2}{c}{ Ropa de casa } & Joyas \\
& $A$ & $B$ & $A$ & $B$ & $A$ & $B$ & $A$ & $B$ & $A$ & $B$ & $A$ & $B$ & $A$ & $B$ \\
\hline-1.500 & 3 & 0,5 & 106 & 19,7 & 49 & 9 & 330 & 61,3 & 51 & 9,5 & 0 & 0 & 0 & \\
$1.500-4.999$ & 237 & 3,3 & 1.560 & 21,7 & 1.041 & 14,5 & 2.889 & 40,3 & 1.412 & 19,7 & 38 & 0,5 & 0 & \\
$5.000-9.999$ & 301 & 3,1 & 1.816 & 18,8 & 1.104 & 11,4 & 3.087 & 32 & 3.097 & 32,1 & 238 & 2,6 & 0 & \\
$10.000-19.999$ & 354 & 2,7 & 2.125 & 16,2 & 1.309 & 10 & 5.402 & 41,3 & 3.575 & 27,3 & 253 & 1,9 & 73 & 0,6 \\
+20.000 & 230 & 3,1 & 1.638 & 22,5 & 1.111 & 15,2 & 1.587 & 21,8 & 2.431 & 33,3 & 256 & 3,5 & 41 & 0,6 \\
\hline
\end{tabular}

A, reales; B, \%

Fuente: A. H.P.L. (45 inventarios con los bienes tasados). 
